\title{
KRAS mutation-induced upregulation of PD-L1 mediates immune escape in human lung adenocarcinoma
}

\author{
Nan Chen ${ }^{1,2,3}$ - Wenfeng Fang ${ }^{1,2} \cdot$ Zhong Lin $^{3} \cdot$ Peijian Peng ${ }^{3}$ Juan Wang ${ }^{3}$. \\ Jianhua Zhan ${ }^{1,2} \cdot$ Shaodong Hong ${ }^{1,2} \cdot$ Jiaxing Huang ${ }^{3} \cdot$ Lin Liu $^{3} \cdot$ Jin Sheng ${ }^{1,2}$. \\ Ting Zhou ${ }^{1,2} \cdot$ Ying Chen ${ }^{3} \cdot$ Hongyu Zhang $^{3} \cdot$ Li Zhang $^{1,2}$
}

Received: 24 July 2016 / Accepted: 18 April 2017 / Published online: 27 April 2017

(C) The Author(s) 2017. This article is an open access publication

\begin{abstract}
It was reported that PD-L1 expression was correlated with genetic alterations. Whether PD-L1 was regulated by mutant Kirsten rat sarcoma viral oncogene homolog (KRAS) in non-small-cell lung cancer (NSCLC) and the underlying molecular mechanism were largely unknown. In this study, we investigated the correlation between PD-L1 expression and KRAS mutation and the functional significance of PD-1/PD-L1 blockade in KRASmutant lung adenocarcinoma. We found that PD-L1 expression was associated with KRAS mutation both in the human lung adenocarcinoma cell lines and tissues. PD-L1 was up-regulated by KRAS mutation through p-ERK but not p-AKT signaling. We also found that KRAS-mediated up-regulation of PD-L1 induced the apoptosis of CD3positive $T$ cells which was reversed by anti-PD-1 antibody (Pembrolizumab) or ERK inhibitor. PD-1 blocker or ERK
\end{abstract}

Electronic supplementary material The online version of this article (doi:10.1007/s00262-017-2005-z) contains supplementary material, which is available to authorized users.

The authors, Nan Chen, Wenfeng Fang, and Zhong Lin contributed equally to this work.

Li Zhang

zhangli@sysucc.org.cn

1 State Key Laboratory of Oncology in South China, Department of Medical Oncology, Sun Yat-Sen University Cancer Center, 651 Dongfeng Road East, Guangzhou, Guangdong, People's Republic of China

2 Collaborative Innovation Center for Cancer Medicine, Sun Yat-sen University Cancer Center, Guangzhou, Guangdong, People's Republic of China

3 Department of Medical Oncology, the Fifth Affiliated Hospital of Sun Yat-sen University, Zhuhai, Guangdong, People's Republic of China inhibitor could recover the anti-tumor immunity of $\mathrm{T}$ cells and decrease the survival rates of KRAS-mutant NSCLC cells in co-culture system in vitro. However, Pembrolizumab combined with ERK inhibitor did not show synergistic effect on killing tumor cells in co-culture system. Our study demonstrated that KRAS mutation could induce PD-L1 expression through p-ERK signaling in lung adenocarcinoma. Blockade of PD-1/PD-L1 pathway may be a promising therapeutic strategy for human KRAS-mutant lung adenocarcinoma.

Keywords KRAS $\cdot$ PD-L1 $\cdot$ PD-1 $\cdot$ Lung adenocarcinoma
Abbreviations
ALK Anaplastic lymphoma kinase
CST Cell signaling technology
CTLA-4 Cytotoxic T lymphocyte associated antigen-4
DC-CIK Dendritic cells and cytokine-induced killer cells
EGFR Epidermal growth factor receptor
EML4
Echinoderm microtubule associated protein like 4
KRAS Kirsten rat sarcoma viral oncogene homolog
NSCLC Non-small-cell lung cancer
PD-1 Programmed death-1 receptor
PD-L1 Programmed death ligand 1
TKIs Tyrosine kinase inhibitors

\section{Introduction}

Lung cancer remains the leading cause of cancer-related death worldwide [1]. Non-small-cell lung cancer (NSCLC) accounts for approximately $80 \%$ of all lung cancers [2]. Lung adenocarcinoma, as the most common pathologic type of NSCLC, is often accompanied with oncogenic 
driver mutation [3, 4]. Driver mutations like epidermal growth factor receptor mutation (EGFR) and echinoderm microtubule associated protein like 4 and anaplastic lymphoma kinase (EML4-ALK) fusion are highly sensitive to their corresponding tyrosine kinase inhibitors (TKIs) [5]. Kirsten rat sarcoma viral oncogene homolog (KRAS) is the most common driver mutation in lung adenocarcinoma patients of non-Asian ethnicity [6]. The prevalence of KRAS mutation in lung adenocarcinoma in Asian and Western patients is approximately 11 and $26 \%$, respectively [7]. In addition, KRAS mutation is usually mutually exclusive with other major driver mutations such as EGFR and ALK [8]. Recent studies show that patients with KRASmutant lung cancer respond poorly to EGFR-TKIs [9, 10]. Furthermore, KRAS mutation is a negative predictor of the efficacy of chemotherapy [11]. Until now, the more effective treatment strategies are urgently needed for KRASmutant NSCLC.

Immune checkpoint molecules, programmed death-1 receptor (PD-1, CD279) and programmed death ligand 1 (PD-L1, B7-H1 or CD274) play an important role in tumor immune escape [12]. Recent development of immune checkpoint inhibitors such as anti-PD-1 antibody and antiCTLA-4 antibody has shown promising results in specific subset of NSCLC patients [13, 14]. Some studies reported that high PD-L1 expression was correlated with EGFR mutation and ALK fusion protein in NSCLC [15-17]. Thus, exploring the association between PD-L1 expression and driver mutations and determining the effect of immune checkpoint inhibitors on oncogene addicted NSCLC are crucial in clinical practice. However, whether PD-L1 is regulated by KRAS and the underlying molecular mechanisms are largely unknown. Moreover, the effect of blocking PD-L1/PD-1 axis on T cells and NSCLC cells and its potential clinical value in KRAS-mutant NSCLC have not been fully elucidated.

In this study, we investigated the correlation between PD-L1 and KRAS mutation and the regulatory mechanism. We also tried to explore whether blocking the PD-1/PD-L1 axis could be a novel therapeutic option for lung adenocarcinoma with KRAS mutation.

\section{Materials/patients and methods}

\section{Cell lines and cell culture}

Human NSCLC cell lines H460, H1299, H2228, H292 and H1993 were obtained from the American Type Culture Collection. Immortalized human lung bronchial epithelial cell (Beas-2B), EKVX, Beas-2B-vector, Beas-2B-KRASG12D and Beas-2B-KRAS-WT cells were generously provided by Prof. Liang Chen (National Institute of Biological
Sciences, China). H358 was kindly provided by Prof. Mengfeng Li (Department of Microbiology, Zhongshan School of Medicine, Sun Yat-sen University, China). H358, H460 and EKVX are the KRAS-mutant NSCLC cell lines. H1299 is the N-RAS-mutant lung adenocarcinoma cell line. H2228 is the lung adenocarcinoma cell line with EML4-ALK fusion. H292 and H1993 are NSCLC cell lines with EGFR/ALK/KRAS wild-type (WT). Beas-2B-KRASG12D, Beas-2B-KRAS-WT and Beas-2B-vector are the Beas-2B cells stably transfected with KRAS G12D mutant, KRAS wild-type and control plasmid, respectively. H2228 and H358 were cultured in RPMI-1640 complete growth medium supplemented with $10 \%$ fetal bovine serum and antibiotics $(10,000 \mathrm{U} / \mathrm{ml}$ penicillin and $10 \mu \mathrm{g} / \mathrm{ml}$ streptomycin). Other cell lines were grown in DMEM complete medium.

\section{Western blot analysis and quantitative real-time PCR}

Western blot analysis was done as previously reported [15]. The primary antibodies for PD-L1 (E1L3N ${ }^{\mathrm{TM}}$ ), RAS (D2C1), mutant KRAS (G12D Mutant Specific) (D8H7), phospho-p44/42MAPK (ERK1/2) (Thr202/Tyr204), p44/42MAPK (ERK1/2), phosphor-AKT (Ser473), AKT and GAPDH were purchased from Cell Signaling Technology (CST). Quantitative real-time PCR experiments were performed as previous described [18].

\section{Surface staining of PD-L1 with flow cytometry}

Suspension cells (Beas-2B-vector, Beas-2B-KRASG12D and Beas-2B-KRAS-WT) were stained with PD-L1 (E1L3N, Rabbit mAb, PE Conjugated) or the corresponding isotype control (DA1E, Rabbit mAb IgG, PE Conjugated) (CST, Danvers, MA). The surface expression of PD-L1 were detected by flow cytometry and analyzed with FlowJo 7.6.1 software [15].

\section{Immunofluorescence}

H358, H1993, Beas-2B-KRAS-G12D and Beas-2B-vector cells were fixed and blocked before addition of the primary antibodies including PD-L1 (E1L3N ${ }^{\mathrm{TM}}$, Rabbit mAb) or mutant KRAS (G12D mutant specific, D8H7, Rabbit mAb) at $4{ }^{\circ} \mathrm{C}$ overnight. Then cells were incubated with secondary antibody (Alexa Fluor 488 or 555 donkey anti-rabbit IgG $[\mathrm{H}+\mathrm{L}]$, Life Technologies, LA) for $1 \mathrm{~h}$. The detailed protocol was described in previous report [15].

\section{KRAS siRNA, inhibitors and cell viability analysis}

KRAS siRNAs were purchased from Ribobio Corporation (Guangzhou, China). The target sequence of KRAS siRNA 
\#1 and siRNA \#2 are CGAATATGATCCAACAATA and CAAGAGGAGTACAGTGCAA, respectively. Beas-2BKRAS-G12D and H358 cells were transiently transfected with KRAS siRNAs using Lipofectamine ${ }^{\circledR}$ RNAiMAX_ Reagent (Invitrogen) for $48 \mathrm{~h}$. ERK1/2 inhibitor (SCH772984) and AKT1/2/3 inhibitor (MK-22062HCL) were purchased from Selleckchem (Houston, USA). Beas2B-KRAS-G12D cells or H358 cells were exposed to climbing doses of ERK and AKT inhibitors for $72 \mathrm{~h}$. The viability of the cells was tested with CCK8 kit (Cell Counting Kit-8, Dojindo.Co, Japan). Recombinant humanized anti-PD-1 antibody, Pembrolizumab (MK-3475, Keytruda) was from Merck Sharp \& Dohme Corp (Whitehouse Station, NJ08889, USA).

\section{Co-culture system and apoptosis assay with flow cytometry}

Dendritic cells and cytokine-induced killer cells (DC-CIK) were kindly provided by Prof. Jianchuan Xia (Department of Biotherapy, Sun Yat-sen University Cancer Center, China) $[19,20]$. The protocol of acquiring DC-CIK was described in our previous report [21]. Beas-2B-KRASG12D, Beas-2B-vector, H358 and EKVX cells were seeded into 12 -well plates at a density of $1.0 \times 10^{5}$ cells/ well, respectively. The acquired DC-CIK were added into co-culture system with Beas-2B-KRAS-G12D, Beas-2Bvector, $\mathrm{H} 358$ or EKVX cells at the ratio of $1: 1$, respectively. Next, the DC-CIK/Beas-2B-KRAS-G12D, DC-CIK/ H358 and DC-CIK/EKVX co-culture system were treated with mock, Pembrolizumab (500 $\mu \mathrm{g} / \mathrm{ml})$, ERK1/2 inhibitor $(100 \mathrm{nM} / \mathrm{L})$ or AKT inhibitor $(1.0 \mu \mathrm{M} / \mathrm{L})$, respectively. After $48 \mathrm{~h}$, suspended DC/CIK cells were removed from the adherent Beas-2B-KRAS-G12D, H358 or EKVX cells in the cell culture plate with pipet. Then, after washing with PBS, suspended DC/CIK cells were stained with anti-human CD3 FITC antibody (OKT-3, 11-0037, Affymetrix eBioscience) for $15 \mathrm{~min}$. Next, after washing, DCCIK cells were stained with Annexin V-APC and 7-AAD for 15 min with Apoptosis Detection kit (KGA1023-1026, KeyGEN, Nanjing, China) [21]. The apoptotic cells of CD3 + $\mathrm{T}$ cells detected by flow cytometry (Beckman Gallios, Beckman-Coulter, Inc. USA) were defined as Annexin V-APC-positive cells (both 7-AAD-negative and -positive) from the gate of CD3-positive cells. The example of gating strategy is presented in supplementary Fig. 1.

\section{Real time cells survival analysis}

The survival rates of KRAS-mutant tumor cells like H358 or EKVX cells were dynamically monitored in real time by the xCELLigence system (E-plate, Roche) which could exclude the interference of suspended DC-CIK. Firstly, 96-well E-plate with $50 \mu 1$ of complete growth medium in each well was tested in the incubator to establish a background reading. Next, tumor cells $\left(1.0 \times 10^{4}\right.$ cells/well $)$ were seeded into 96-well E-plates for approximately $20 \mathrm{~h}$ followed by addition of DC-CIK $(50 \mu \mathrm{l} / \mathrm{well})$ into the E-plates at a DC-CIK: tumor cells ratio of 1:1. Finally, an additional $50 \mu \mathrm{l} /$ well of the complete medium containing different drugs such as vehicle, Pembrolizumab $(500 \mu \mathrm{g} /$ $\mathrm{ml})$, ERK1/2 inhibitor (100 nM/L) and Pembrolizumab $(500 \mu \mathrm{g} / \mathrm{ml})$ plus ERK1/2 inhibitor $(100 \mathrm{nM} / \mathrm{L})$ were added into the DC-CIK/H358 or DC-CIK/EKVX co-culture system, respectively. $\mathrm{H} 358$ cells alone were meanwhile treated with vehicle, Pembrolizumab $(500 \mu \mathrm{g} / \mathrm{ml})$ and ERK1/2 inhibitor $(100 \mathrm{nM} / \mathrm{L})$ as the control groups. Cell index values were monitored every $15 \mathrm{~min}$ from each well of E-plate and presented as the dynamic cell growth curves [21, 22].

\section{Patients and clinical data}

Our study prospectively enrolled 216 newly diagnosed NSCLC patients who all underwent genomic analysis of EGFR, ALK and KRAS from April 2013 to December 2014 in Sun Yat-sen University Cancer Center (SYSUCC). This study was approved by the Institutional Review Board of SYSUCC and written informed consent was obtained before specimens were collected. The specimens were from surgical resection tissue or biopsies of the untreated patients. KRAS and EGFR mutation status were tested using real-time PCR. ALK rearrangements were detected by fluorescence in situ hybridization. Excluding the patients with EGFR mutation and ALK fusion, the remaining 69 patients were pathologically diagnosed as lung adenocarcinoma with EGFR/ALK wild-type. Among them, there were 19 patients harboring KRAS mutation. Patients' baseline characteristics were collected including gender, age, smoking status, tumor differentiation and staging. Pathologic or clinical staging was determined according to the cancer staging manual ( 7 th edition) of American Joint Committee on Cancer. Using "MatchIt" package of $\mathrm{R}$ programming language, baseline characteristics of patients were balanced matching between KRAS mutation group and EGFR/ALK/KRAS wild-type group by propensity matching score analysis [23]. Subsequently, statistic analysis has been carried out for 19 patients with KRAS mutation matched with 38 out of 50 patients with EGFR/ALK/KRAS wild-type. Finally, PD-L1 expression in the tissue of 57 patients after matching was detected by immunohistochemistry.

\section{Immunohistochemistry}

Immunohistochemical staining was performed using PD-L1 rabbit antibody (E1L3N ${ }^{\mathrm{TM}}$, CST; dilution 1:200) 
a

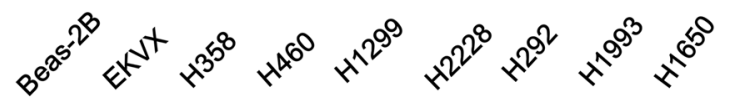

PD-L1

GAPDH

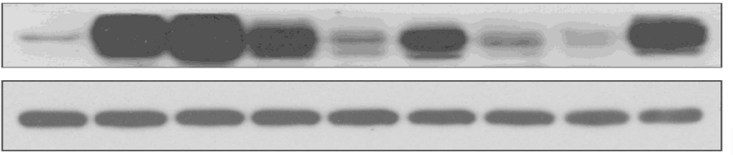

C
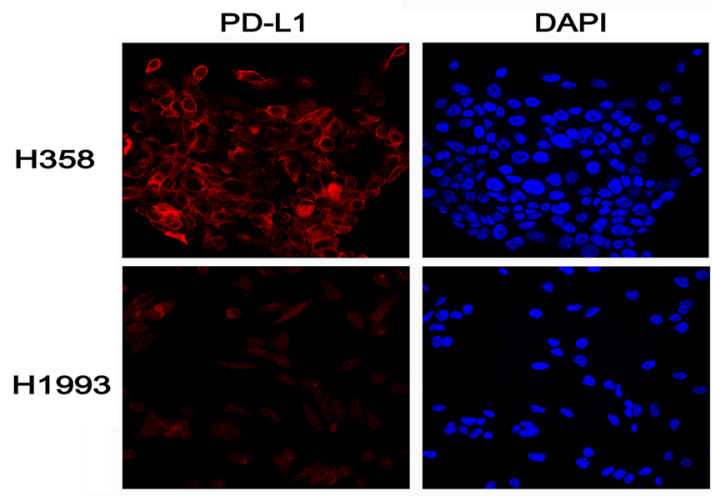

e

KRAS mutation case
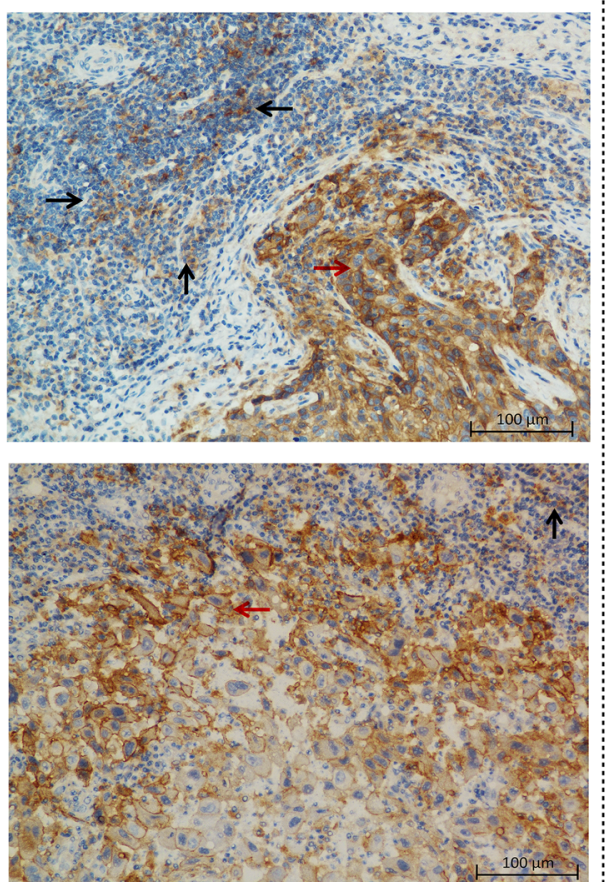

overnight at $4{ }^{\circ} \mathrm{C}$. Immunoreactivity was detected using the DAKO ChemMateEnVision method according to the manufacturer's instructions. Two pathologists blinded to patients' information independently assessed expression b

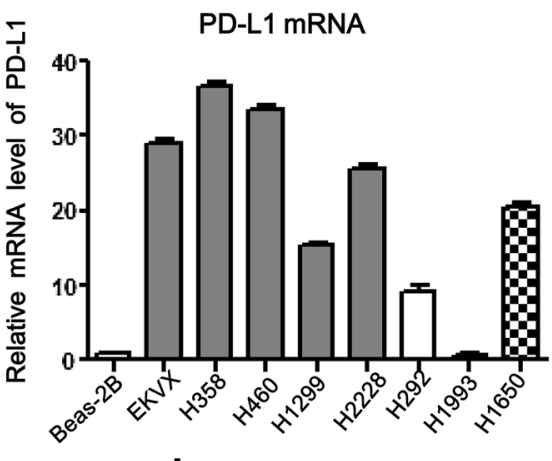

d
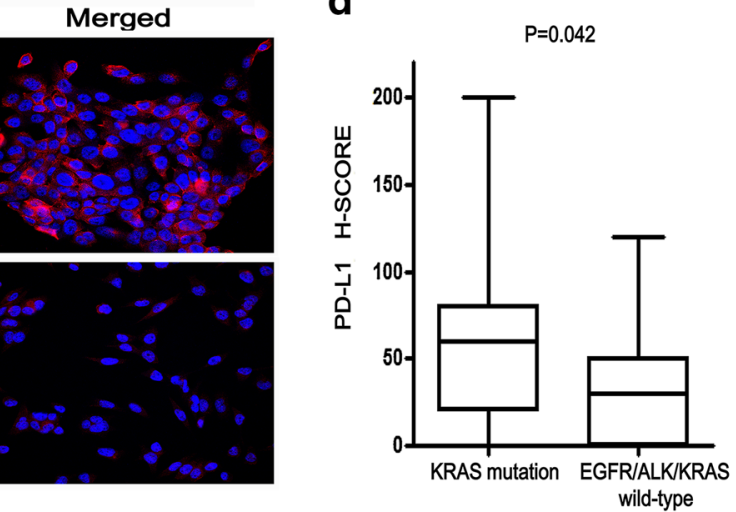

EGFR/ALK/KRAS

wild-type case
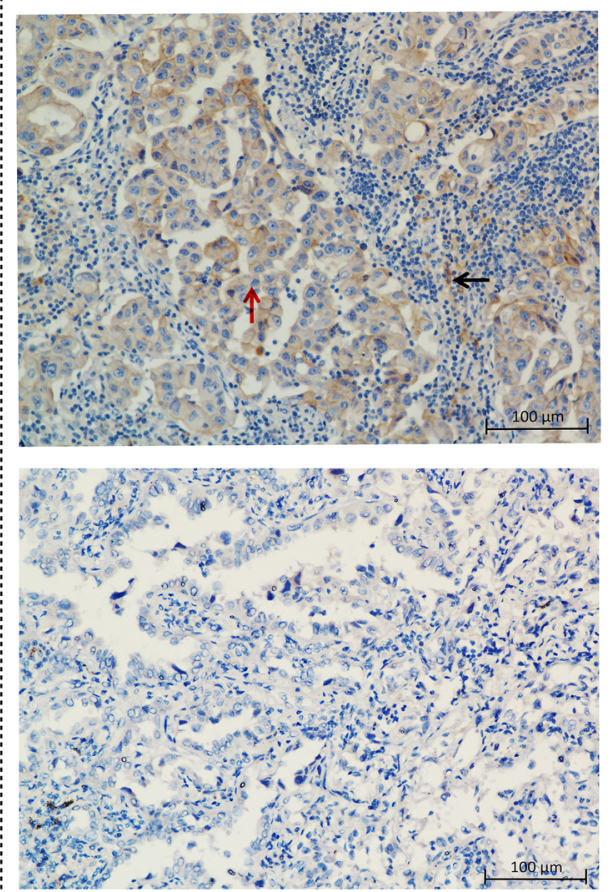

of PD-L1. Semi-quantitative H score (H-SCORE) was determined by multiplying the percentage of positively stained cells by an intensity score ( 0 , absent; 1 , weak; 2 , moderate; and 3, strong) and ranged 0-300. 
\Fig. 1 PD-L1 expression correlated with KRAS mutation. a The protein expression levels of PD-L1 were detected by western blot in different NSCLC cell lines and Beas-2B. GAPDH was used as loading reference. $\mathbf{b}$ The relative expression levels of PD-L1 mRNA were detected by real time PCR in above-mentioned cells. $\mathbf{c}$ The localization of PD-L1 (red signal) in H358 and H1993 cell lines were shown by immunofluorescence counterstained with DAPI (blue signal). Original magnification: $\times 600$. d Significant association of PD-L1 H-SCORE with KRAS status (19 cases of lung adenocarcinoma with KRAS mutation and 38 cases of lung adenocarcinoma with EGFR/ALK/KRAS wild-type). Data are presented as box plots, and $P$ values were determined with the Wilcoxon rank-sum test. e Representative images of PD-L1 immunohistochemical staining in two KRAS-mutant cases with strong staining intensity (left panels) and two EGFR/ALK/KRAS wild-type cases with weak staining intensity (right panels). Black arrows indicate tumor-infiltrating immune cells. Red arrows indicate tumor cells. Original magnification: $\times 400$

\section{Statistical analysis}

The SPSS software (version 19.0) was used for statistical analysis. After matching with "MatchIt" package of R programming language, the differences of gender, smoking status, tumor differentiation, staging between KRAS mutation group and EGFR/ALK/KRAS wild-type group were examined by the Pearson Chi-square test and the difference of age between the two groups was examined by two independent samples' $t$ test. Wilcoxon rank-sum test was used to compare the H-SCORE of PD-L1 staining between KRAS mutation and EGFR/ALK/KRAS wild-type group. Representative results from three independent experiments were shown in this study. Numerical data were presented as the mean \pm standard deviation of the mean (SD). The $P$ values between two experimental groups were tested by two-tailed Student's $t$ test and $P$ values less than 0.05 were considered significant.

\section{Results}

\section{PD-L1 expression was correlated with KRAS mutation in lung adenocarcinoma}

In order to investigate the association between PD-L1 expression and KRAS mutation status, we conducted experiments in human lung adenocarcinoma cell lines and tissue. We found that the protein levels of PD-L1 in endogenous KRAS-mutant NSCLC cell lines (EKVX, H358, H460), EML4-ALK fusion cell line (H2228), and EGFR 19-exon deletion mutation cell line (H1650) were significantly higher than that in EGFR/ALK/KRAS wild-type cell lines (H292, H1993), N-RAS-mutant cell line (H1299), or lung bronchial epithelial cell line (Beas-2B cell) (Fig. 1a). Similar results of PD-L1 mRNA level were also observed in the above-mentioned cell lines (Fig. 1b). Sub-cellular localization of PD-L1 protein was detected in H358 and
H1993 cells using immunofluorescence. PD-L1 showed stronger membrane localization in H358 cells (red fluorescence), compared with that in H1993 cells (Fig. 1c). Next, we detected PD-L1 expression on tumor cells and tumor-infiltrating immune cells in patients' lung adenocarcinoma tissue by immunohistochemistry. Baseline characteristics of patients including gender, age, smoking history, tumor differentiation and stage were balanced matching between KRAS mutation group and EGFR/ALK/KRAS wild-type group by propensity matching score analysis [24] (Table 1). KRAS-mutant cases tended to have higher intensity of PD-L1 staining than EGFR/ALK/KRAS wildtype cases. PD-L1 immunoreactivity was detected mainly on the cytomembrane or slightly in the cytoplasm (or both) of tumor cells or tumor-infiltrating immune cells (Fig. 1e). The median of H-SCORE was significantly higher in KRAS mutation cases than in EGFR/ALK/KRAS wildtype cases (60 vs. $30, P=0.042$ ) (Fig. 1d). The results implied that PD-L1 expression was associated with KRAS mutation in lung adenocarcinoma.

\section{Over-expression or knockdown of KRAS altered PD-L1 expression}

Firstly, we found the expression of PD-L1 was significantly higher in Beas-2B-KRAS-G12D cells compared with Beas-2B-vector cells. However, the expression of PD-L1 in Beas-2B-KRAS-WT cell was not obviously increased compared with control cells. Likely, the phosphorylated expression of ERK1/2 and AKT were activated by mutant KRAS-G12D but not wild-type KRAS (Fig. 2a). Similarly, flow cytometric analysis showed the surface expression of PD-L1 in Beas-2B cells with KRAS G12D mutation was higher than that in Beas-2B cells with KRAS wild-type and vector control (Fig. 2b). The induction of PD-L1 by overexpression of exogenous KRAS was further confirmed at the mRNA level by PCR. The higher level of PD-L1 mRNA was observed in Beas-2B-KRAS-G12D cells than that in Beas-2B-vector and Beas-2B-KRAS-WT cells (Fig. 2c). Immunofluorescence staining also showed the association between KRAS and PD-L1 protein expression. Increased expression of KRAS and PD-L1 was observed on the cytomembrane of Beas-2B cells with KRAS G12D over-expression (Fig. 2d). In contrast, knocking down KRAS expression using two loci KRAS siRNAs decreased the expression level of Ras in Beas-2B-KRAS G12D cells. PD-L1 was also significantly down-regulated following KRAS knockdown (Fig. 2e). Similarly, in H358 cells, the protein expression and mRNA level of PD-L1 were both significantly down-regulated following the knockdown of KRAS by siRNAs (Fig. 2f, g). Taken together, our results demonstrated that over-expression of exogenous KRAS induced PD-L1 expression and knocking down KRAS 
Table 1 Baseline characteristics of lung adenocarcinoma patients

\begin{tabular}{|c|c|c|c|c|}
\hline Characteristics & $\begin{array}{l}\text { Total, } \\
n=57\end{array}$ & $\begin{array}{l}\text { KARS mutation, } n=19 \text { (\% with kras } \\
\text { mutation }\end{array}$ & $\begin{array}{l}\text { EGFR/ALK/KRAS wild type, } n=38 \\
\text { ( } \% \text { with wild type })\end{array}$ & $P$ value after matching \\
\hline \multicolumn{4}{|l|}{ Gender } & \multirow[t]{3}{*}{0.838} \\
\hline Female & 17 & $6(31.6 \%)$ & $11(28.9 \%)$ & \\
\hline Male & 40 & $13(68.4 \%)$ & $27(71.1 \%)$ & \\
\hline \multicolumn{4}{|l|}{ Age, years } & \multirow[t]{3}{*}{0.838} \\
\hline$<60$ & 25 & $9(47.4 \%)$ & $16(42.1 \%)$ & \\
\hline$\geq 60$ & 32 & $10(52.6 \%)$ & $22(57.9 \%)$ & \\
\hline \multicolumn{4}{|l|}{ Smoking history } & \multirow[t]{3}{*}{0.703} \\
\hline Smokers & 34 & $12(63.2 \%)$ & $22(57.9 \%)$ & \\
\hline Non-smokers & 23 & $7(36.8 \%)$ & $16(42.1 \%)$ & \\
\hline \multicolumn{4}{|c|}{ Tumor differentiation } & \multirow[t]{4}{*}{0.764} \\
\hline Poor & 22 & $7(36.8 \%)$ & $15(39.5 \%)$ & \\
\hline Moderate & 31 & $10(52.6 \%)$ & $21(55.3 \%)$ & \\
\hline Well & 4 & $2(10.5 \%)$ & $2(5.3 \%)$ & \\
\hline \multicolumn{4}{|l|}{ Stage } & \multirow[t]{5}{*}{1.000} \\
\hline I & 27 & $9(47.4 \%)$ & $18(47.4 \%)$ & \\
\hline II & 12 & $4(21.1 \%)$ & $8(21.1 \%)$ & \\
\hline IIIA & 9 & $3(15.8 \%)$ & $6(15.8 \%)$ & \\
\hline IIIB-IV & 9 & $3(15.8 \%)$ & $6(15.8 \%)$ & \\
\hline
\end{tabular}

reduced PD-L1 expression. PD-L1 expression level was positively correlated with KRAS mutation.

\section{KRAS up-regulated PD-L1 through p-ERK but not p-AKT signaling}

In order to investigate how KRAS regulate PD-L1 expression, firstly, we examined the two key downstream proteins of Ras pathway (p-ERK and p-AKT) by western blot. Figure 2a showed both p-ERK1/2 and p-AKT were activated by KRAS G12D mutation. Then, we used the inhibitors of ERK1/2 and AKT to block the downstream pathways of KRAS in Beas-2B cells with exogenous expression of KRAS G12D mutation and H358 cell with endogenous expression of KRAS G12C mutation. We found that ERK1/2 inhibitor (SCH772984) inhibited the p-ERK, which further resulted in the decrease of PD-L1 expression (Fig. 3a, c). However, AKT inhibitor (MK-2206 2HCL) could effectively suppress p-AKT, but did not alter the expression level of PD-L1 (Fig. 3b, d). To investigate whether PD-L1 is regulated through the mechanism of specific signaling pathway or the effect of cell viability, we performed the viability analysis of H358 and Beas-2BKRAS G12D cells to find that the indicated concentration of ERK inhibitor and AKT inhibitor only weakly inhibited the viability of the two cells (Fig. 3e, f). Thus, PD-L1 regulated by ERK1/2 signaling is dependent on signaling pathway mechanism. Collectively, KRAS mutation induced
PD-L1 expression through p-ERK1/2 signaling pathway but not p-AKT pathway.

KRAS could induce the apoptosis of $T$ cells through PD-1/PD-L1 axis and blocking PD-1/PD-L1 could reverse the apoptosis of $T$ cells in co-culture system

PD-L1 could lead to apoptosis of T cells, and anti-PD-1 antibody could reverse this process. To investigate whether up-regulation of PD-L1 mediated by KRAS could lead to the apoptosis of T cells, we firstly constructed the stable cell line with KRAS G12D over-expression (Beas2B-KRAS-G12D) (Fig. 4a). DC-CIK was prepared from peripheral blood donated by healthy volunteers and co-cultured with Beas-2B-KRAS-G12D cells or Beas-2B-vector cells at the ratio of $1: 1$. As shown in Fig. 4b, e, the apoptosis rates of $\mathrm{CD} 3+\mathrm{T}$ cells co-cultured with Beas-2B-KRAS G12D cells were higher than that with Beas-2B-vector cells $(21.40 \pm 1.33$ vs. $7.07 \pm 0.09 \%, P=0.0004)$. On the contrary, blockade of PD-1 on T cells with Pembrolizumab in Beas-2B-KRAS G12D/DC-CIK co-culture system reduced the apoptosis rates of $\mathrm{CD} 3+\mathrm{T}$ cells to $8.00 \pm 0.36 \%$ $(P=0.0006)$. Next, we used ERK inhibitor to investigate whether it could reverse the apoptosis of $\mathrm{T}$ cells through inhibiting the expression of PD-L1. As expected, the apoptosis rates of $\mathrm{CD} 3+\mathrm{T}$ cells decreased from $21.40 \pm 1.33$ to $16.03 \pm 0.27 \%(P=0.0167)$. However, AKT inhibitor could not reduce the apoptosis rates of $\mathrm{T}$ cells compared 
a

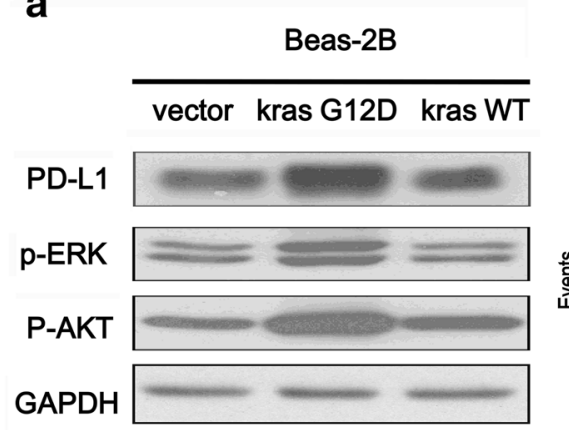

d b

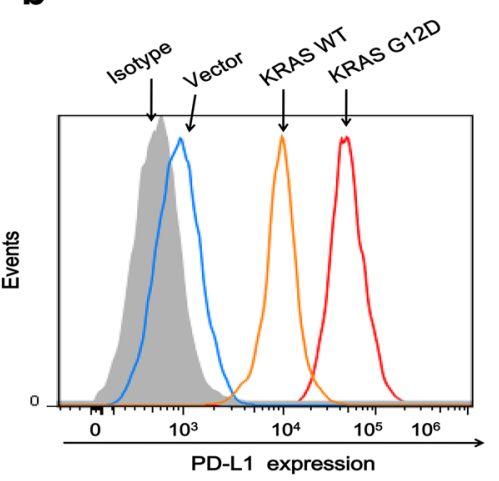

c

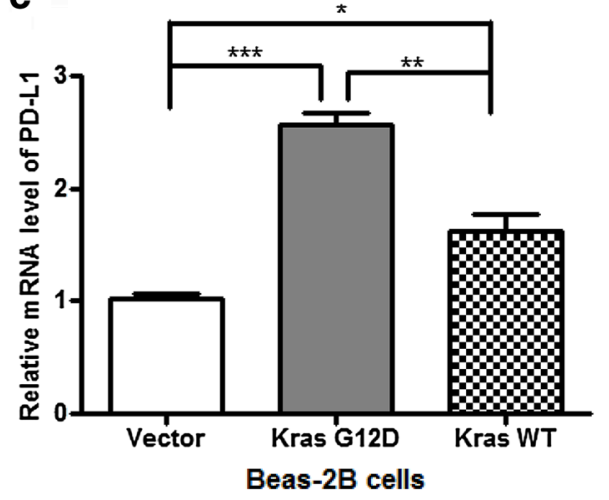

Beas-2B

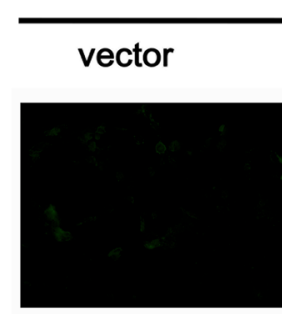

DAPI
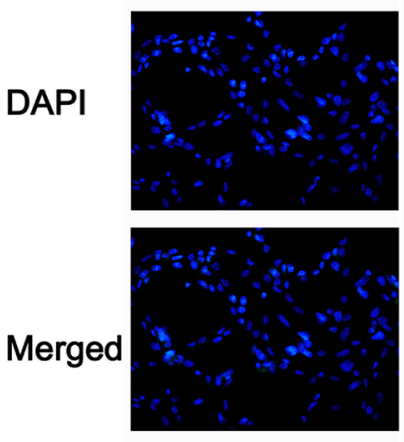

e

Beas-2B-Kras G12D

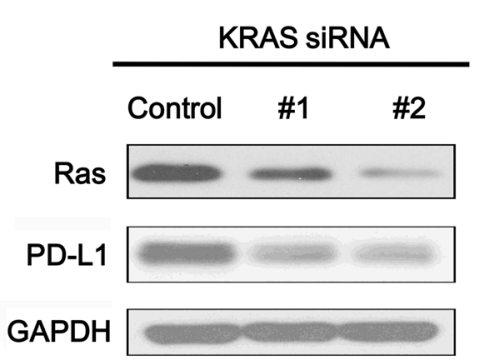

H358

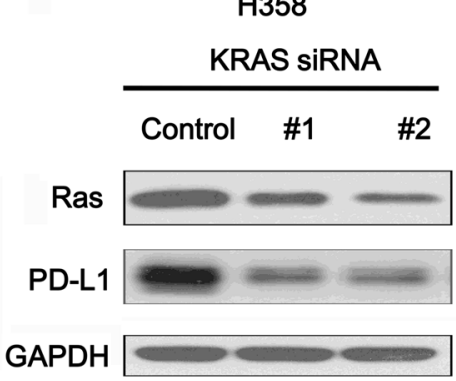

\section{Kras G12D}
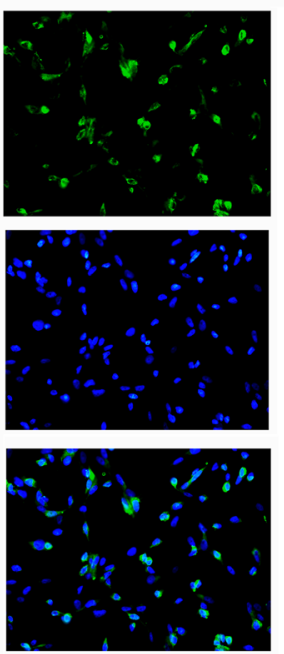

PD-L1

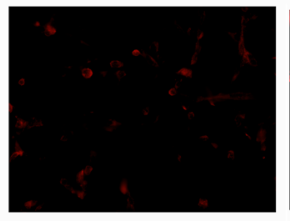

DAPI
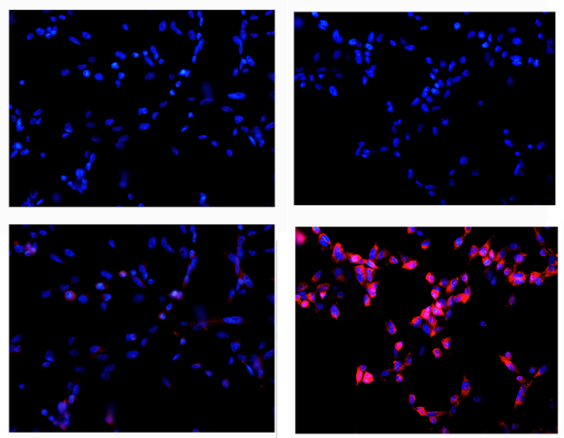

g

\section{Kras G12D}
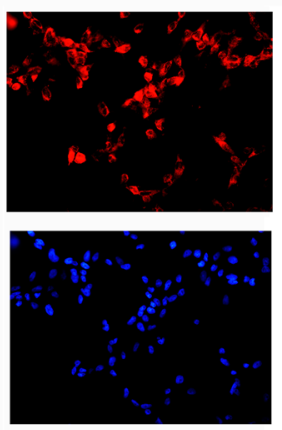

Merged

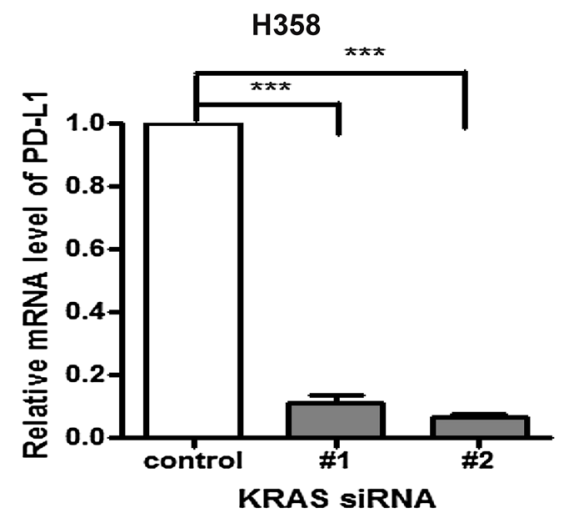

Fig. 2 Both over-expression and knockdown of KRAS regulated PD-L1 expression. a The protein expression level of PD-L1, p-ERK and p-AKT in Beas-2B cells stably transfected with KRAS-G12D mutation, KRAS wild-type and control plasmid, respectively. b The surface expression level of PD-L1 were detected by flow cytometry in Beas-2B-KRAS-G12D, Beas-2B-KRAS-WT, Beas-2B-vector and isotype control cells. $\mathbf{c}$ The relative mRNA level of PD-L1 in Beas2B-KRAS-G12D, Beas-2B-KRAS-WT and Beas-2B-vector cells. d The localization of PD-L1 (red signal) and KRAS (green signal) in Beas-2B-vector and Beas-2B-KRAS-G12D cells were shown by immunofluorescence counterstained with DAPI (blue signal). Original magnification: $\times 200$. e, $\mathbf{f}$ The protein expression level of Ras and PD-L1 were detected by western blot in Beas-2B-KRAS G12D and $\mathrm{H} 358$ cells which were both transiently transfected with two loci KRAS-siRNAs for $48 \mathrm{~h}$. $g$ The relative mRNA level of PD-L1 in H358 cells which were transiently transfected with two loci KRASsiRNAs for $48 \mathrm{~h}$. Representative data of three independent experiments are shown. $* P<0.05 ; * * P<0.001 ; * * * P<0.0001$ 
a

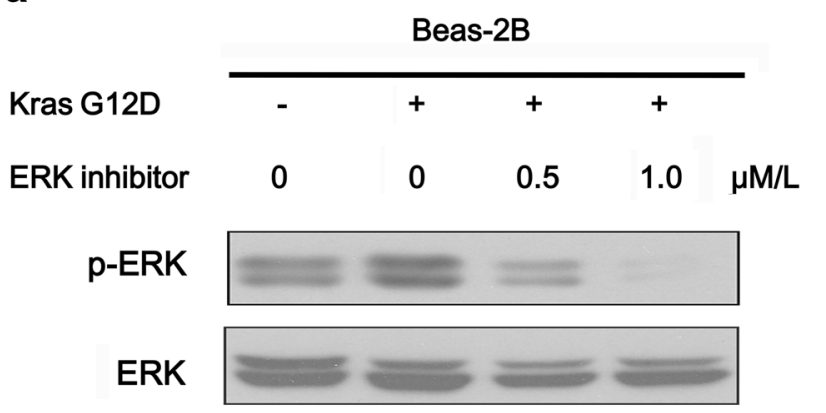

PD-L1

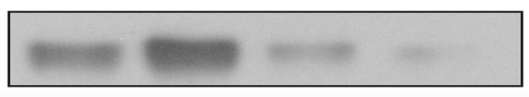

GAPDH

C

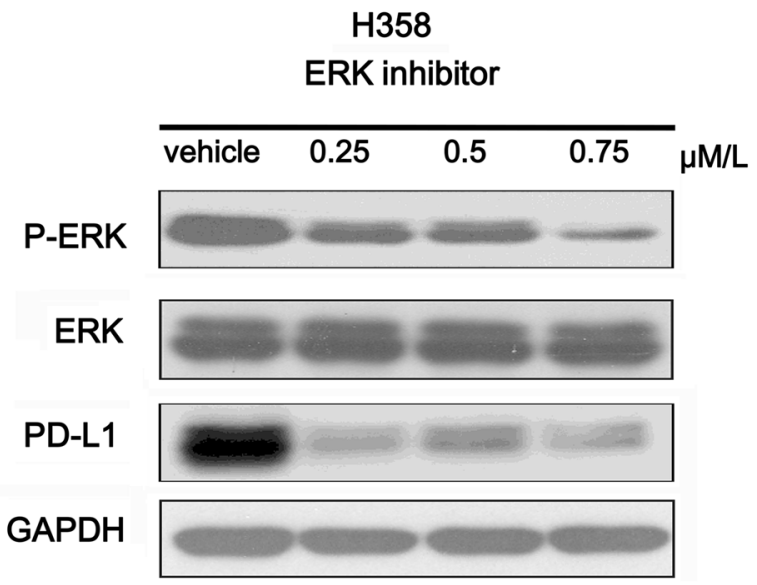

e

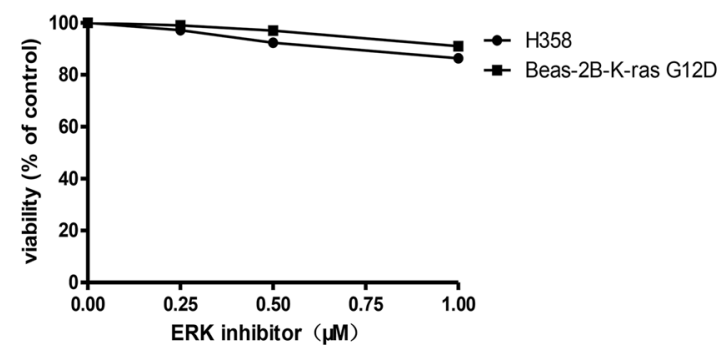

Fig. 3 KRAS regulated PD-L1 through p-ERK but not p-AKT signaling. a The protein expression level of p-ERK, ERK, PD-L1, GAPDH in Beas-2B-vector cells and Beas-2B-KRAS-G12D cells which were treated with $0,0.5,1.0 \mu \mathrm{M}$ ERK1/2 inhibitor (SCH772984) for $48 \mathrm{~h}$. b The protein expression level of p-AKT, AKT, PD-L1, GAPDH in Beas-2B-vector cells and Beas-2B-KRAS G12D cells which were treated with $0,1.0,2.0 \mu \mathrm{M} / \mathrm{L}$ AKT inhibitor (MK-2206 2HCL) for $48 \mathrm{~h}$. c The protein expression level of p-ERK, ERK, PD-L1 and GAPDH in H358 cell which were treated with 0, b

\begin{tabular}{|c|c|c|c|c|}
\hline Kras G12D & - & + & + & + \\
\hline AKT inhibitor & 0 & 0 & 1.0 & 2.0 \\
\hline
\end{tabular}

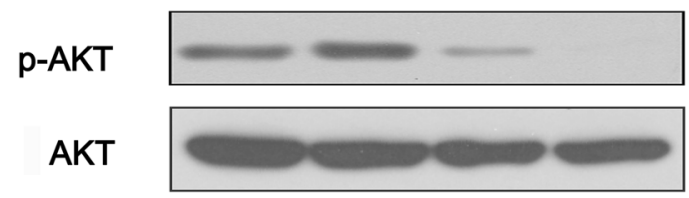

PD-L1

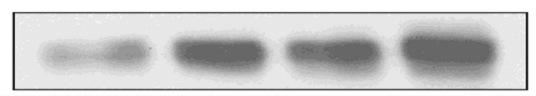

GAPDH

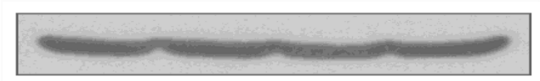

H358

AKT inhibitor

\begin{tabular}{llll}
\hline vehicle & 0.5 & 1.0 & 2.0
\end{tabular} $\mathrm{MM} / \mathrm{L}$

P-AKT

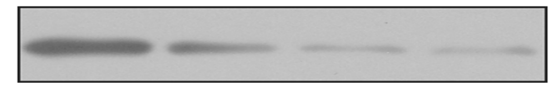

AKT

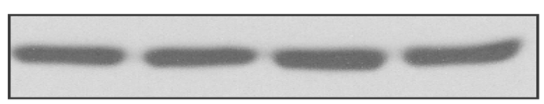

PD-L1

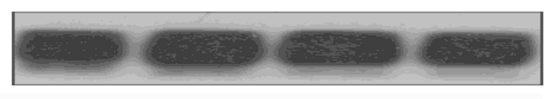

GAPDH

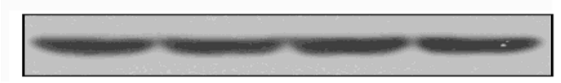

$\mathbf{f}$

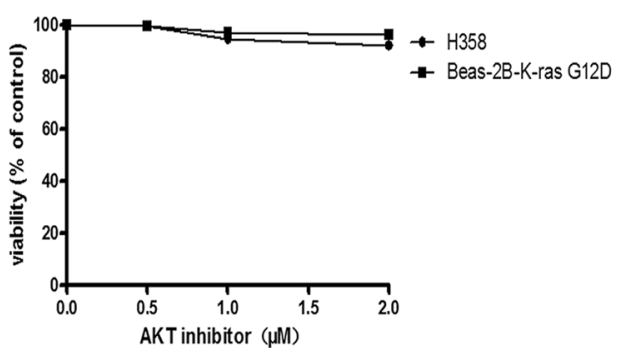

$0.25,0.5,0.75 \mu \mathrm{M} / \mathrm{L}$ ERK1/2 inhibitor (SCH772984) for $48 \mathrm{~h}$. d The protein expression level of p-AKT, AKT, PD-L1 and GAPDH in $\mathrm{H} 358$ cell which were treated with $0,0.5,1.0,2.0 \mu \mathrm{M}$ AKT inhibitor (MK-2206 2HCL) for $48 \mathrm{~h}$. Vehicle represented PBS. e, f Cell viability of $\mathrm{H} 358$ cells and Beas-2B-KRAS G12D cells were detected with CCK8 kit when they were treated with climbing doses of ERK 1/2 inhibitor $(0,0.25,0.75,1.0 \mu \mathrm{M} / \mathrm{L})$ and AKT inhibitors $(0,0.5 .1 .0$, $2.0 \mu \mathrm{M} / \mathrm{L}$ ) for $72 \mathrm{~h}$. The experiments were repeated three times. Representative data are shown 
a

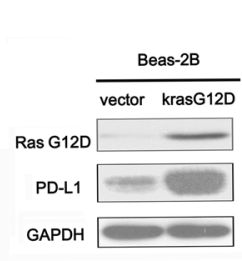

e b

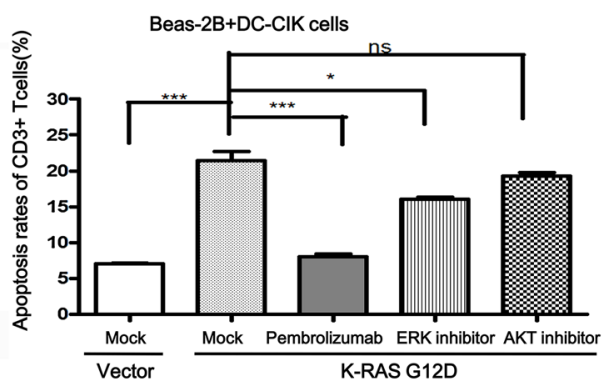

C

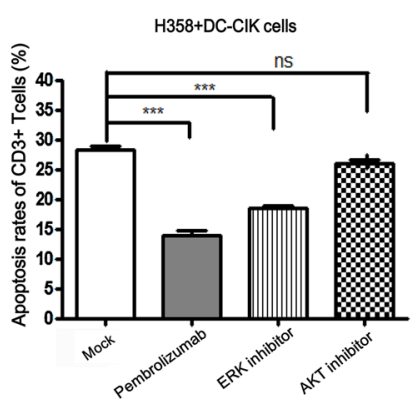

d

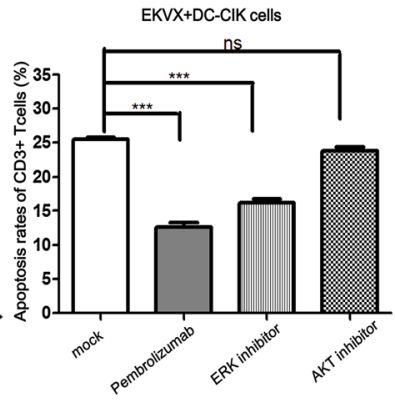

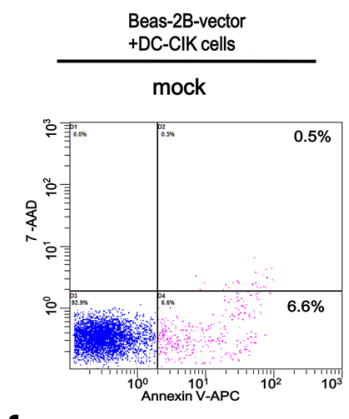

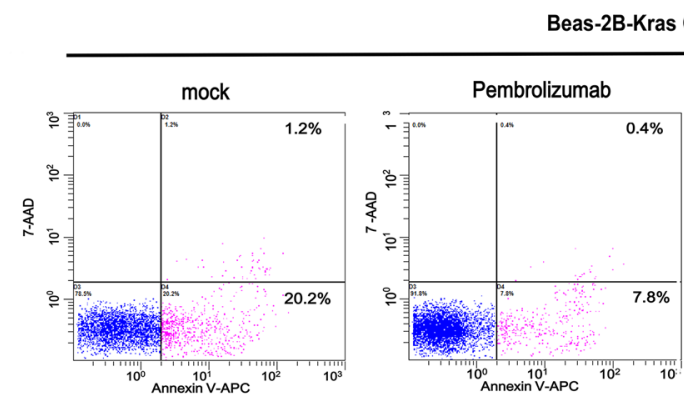

Beas-2B-Kras G12D+DC-ClK cells

f

H358+DC-CIK cells

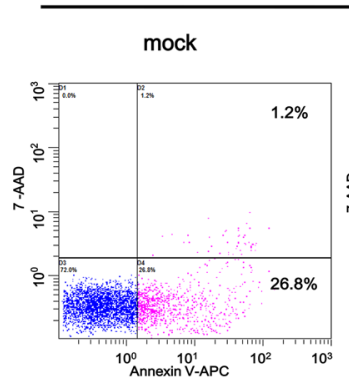

g
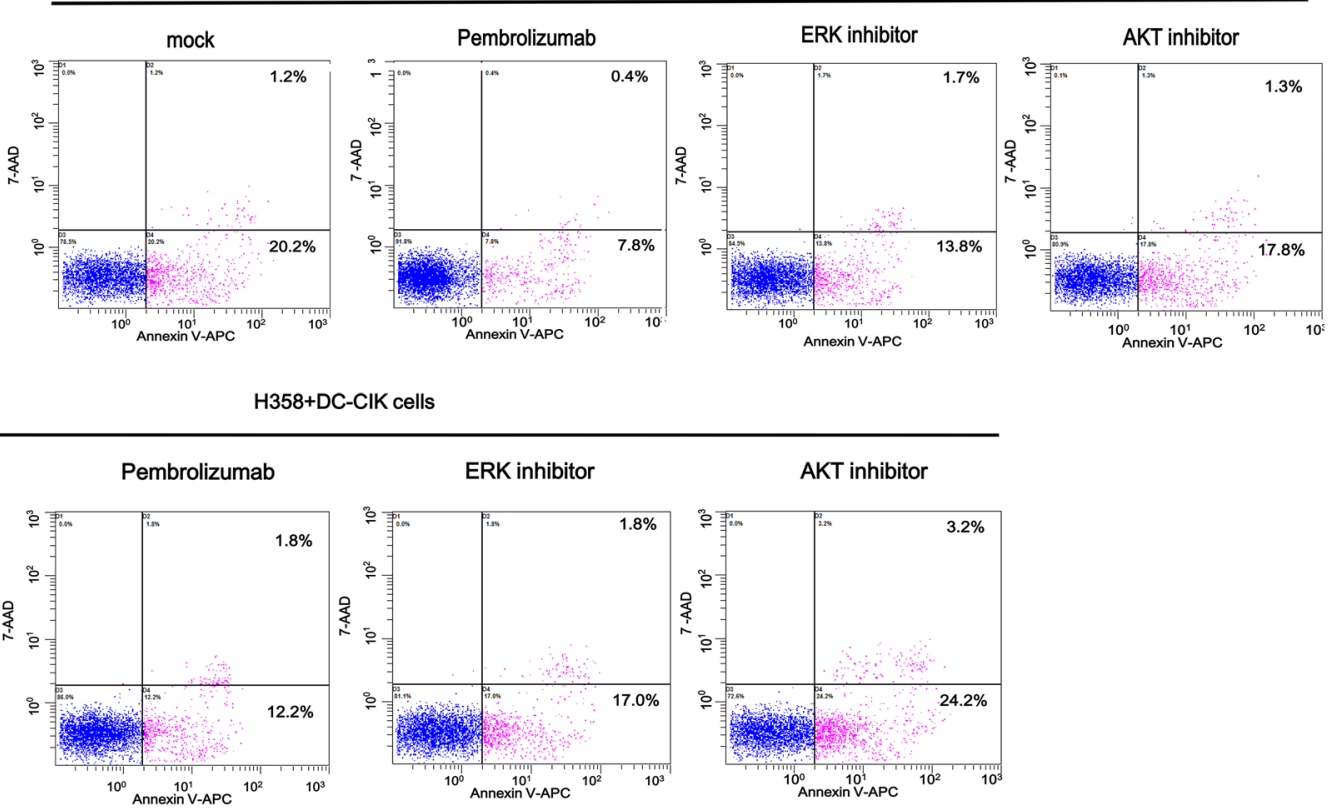

EKVX+DC-CIK cells
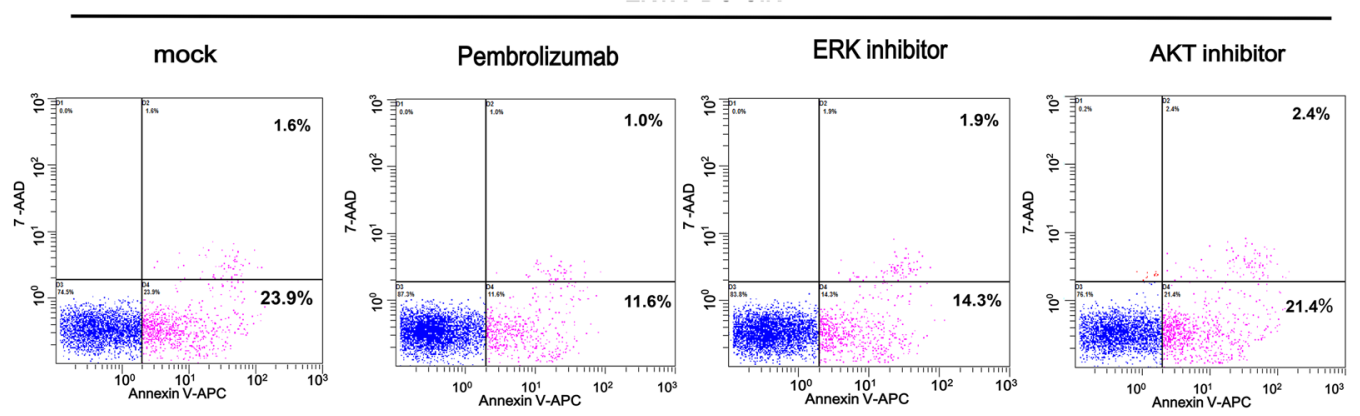

Fig. 4 KRAS induced the apoptosis of CD3+ T cells through PD-L1/PD-1 axis and blocking PD-1/PD-L1 could reverse the process. a The protein expression level of KRAS-G12D and PD-L1 in Beas-2B-vector and Beas-2B-KRAS G12D cells were detected by western blot. Vector represented the control plasmid of KRAS G12D. Fig. b, c, $\mathbf{d}$ is the statistical histogram of Fig. e, f, g. The apoptosis rates of $\mathrm{CD} 3+\mathrm{T}$ cells were detected by Annexin V-APC/7-AAD apoptosis assay in DC-CIK co-cultured with Beas-2B-vector cells or Beas-2B-KRAS G12D cells (e), DC-CIK/H358 co-culture system (f) or DC-CIK/EKVX co-culture system (g) which were respectively treated with mock (negative control), Pembrolizumab $(500 \mu \mathrm{g} /$ $\mathrm{ml})$, ERK1/2 inhibitor (100 nM/L) and AKT inhibitor $(1.0 \mu \mathrm{M} / \mathrm{L})$. The Annexin V-APC-positive cells (both 7-AAD-negative and -positive) were defined as apoptotic cells. Representative results from three independent experiments are shown. $* P<0.05 ; * * P<0.001$; $* * * P<0.0001 ; n s$ indicated as no statistical significance 
with mock group $(19.23 \pm 0.52$ vs. $21.40 \pm 1.33 \%$, $P=0.2037)$. Next, in H358/DC-CIK co-culture system, ERK inhibitor also reduced the apoptosis rates of CD3+ $\mathrm{T}$ cells from $28.30 \pm 0.62$ to $18.67 \pm 0.30 \%(P=0.0002$, Fig. 4c, f). However, the apoptosis rates of $\mathrm{CD} 3+\mathrm{T}$ cells in the AKT inhibitor group and mock group did not differ $(26.10 \pm 0.65$ vs. $28.30 \pm 0.62 \%, P=0.0713)$. We also found that the apoptosis rates of $\mathrm{CD} 3+\mathrm{T}$ cells decreased from $28.30 \pm 0.62 \%$ in the mock group to $14.03 \pm 0.84 \%$ in the Pembrolizumab group $(P=0.0002)$. Similarly, we chose EKVX cells (another KRAS-mutant NSCLC cell line) to further confirm that ERK inhibitor and Pembrolizumab could reduce the apoptosis rates of $\mathrm{CD} 3+\mathrm{T}$ cells $(25.47 \pm 0.32$ vs. $16.17 \pm 0.61 \%, P=0.0002$ and $25.47 \pm 0.32$ vs. $12.60 \pm 0.64 \%, P<0.0001)$ in EKVX/ DC-CIK co-culture system but not with AKT inhibitor ( $25.47 \pm 0.32$ vs. $23.83 \pm 0.55 \%, P=0.0616$ ) (Fig. 4 d, g). To understand whether the inhibitors and Pembrolizumab may directly affect the $\mathrm{T}$ cells, we tested the effects of the two inhibitors and Pembrolizumab on $\mathrm{T}$ cells alone. We found that Pembrolizumab $(500 \mu \mathrm{g} / \mathrm{ml})$, ERK1/2 inhibitor $(100 \mathrm{nM} / \mathrm{L})$ and AKT inhibitor $(1 \mu \mathrm{M} / \mathrm{L})$ could not influence the viability of DC-CIK and the apoptosis of CD3+ $\mathrm{T}$ cells (Supplementary Fig. 2).Taken together, our results demonstrated that KRAS-mediated up-regulation of PD-L1 could induce the apoptosis of CD3 + T cells through PD-1/ PD-L1 axis while blocking PD-1 or inhibiting ERK pathway could alleviate the apoptosis of $\mathrm{CD} 3+\mathrm{T}$ cells.

\section{Blocking PD-1/PD-L1 axis decreased the survival of KRAS-mutant cells of lung adenocarcinoma in co-culture system}

Further we examined the real-time survival signal of attached tumor cells after blockade of PD-1 by anti-PD-1 antibody or inhibiting PD-L1 by ERK inhibitor in tumor cells/DC-CIK co-culture system. The cell index represents the survival rate of attached tumor cells excluding the interference of suspended DC-CIK. When H358 cells alone was treated with Pembrolizumab $(500 \mu \mathrm{g} / \mathrm{ml})$, ERK inhibitor $(100 \mathrm{nM} / \mathrm{L})$ or vehicle, the cell index of $\mathrm{H} 358$ was not obviously changed. However, when H358 or EKVX cells, respectively, co-cultured with DC-CIK at the ratio of 1:1, the survival rates of H358 and EKVX cells decreased in the presence of Pembrolizumab $(500 \mu \mathrm{g} / \mathrm{ml})$, ERK inhibitor $(100 \mathrm{nM} / \mathrm{L})$ or both. But combined treatment of Pembrolizumab and ERK inhibitor did not show synergistic tumor-suppression effect (Fig. 5a, b). These results demonstrated that anti-PD-1 antibody or ERK inhibitor could recover the anti-tumor immunity of $\mathrm{T}$ cells and therefore decrease the survival rates of KRAS-mutant cells of lung adenocarcinoma in co-culture system.

\section{Discussion}

At present, increasing evidences show that immune suppressive microenvironment is involved in the progression of tumor. The PD-1/PD-L1 axis is an important immune inhibitory pathway contributing to immune escape of cancer cells. It is reported that oncogenes and multiple proinflammatory molecules could regulate PD-L1 [25]. PD-L1 over-expression is more frequently observed in oncogene-addicted lung adenocarcinoma, especially with coexisting mutation subtypes [26]. Our previous studies showed EGFR mutation and ALK rearrangement were associated with PD-L1 expression [15, 16, 27]. Whether PD-L1 was regulated by other driver mutation in NSCLC and its molecular mechanism were largely unknown. In the present study, we demonstrated that PD-L1 expression was positively correlated with KRAS mutation both in the cell lines and tissue of lung adenocarcinoma.

KRAS mutations are detected in approximately $20-25 \%$ of lung adenocarcinoma and $4 \%$ of squamous cell lung carcinoma [4, 8]. Here, we found a greater proportion of KRAS mutation subgroup showed the higher expression of PD-L1, compared with that of EGFR/ALK/KRAS wildtype subgroup in lung adenocarcinoma patients. However, Calles A et al. recently stated PD-L1 expression was not genetically driven by KRAS mutation but induced by smoking [28]. The explanations for this discrepancy are: firstly, the sensitivity and specificity of anti-PD-L1 antibody affects the expression intensity of PD-L1 in immunohistochemistry experiment. We employed E1L3N clone to evaluate PD-L1 expression not only on tumor cells but also on tumor-infiltrating immune cells, whereas they used clone 9A11 to examine PD-L1 only on tumor cells. Mahoney et al. have reported clone E1L3N may be more sensitive than clone 9A11 in immunohistochemistry [29]. Moreover, the two studies used different cohorts of Eastern and Western patients. Racial difference may play a crucial role in the controversial results. Similar to our finding, recent research of Skoulidis demonstrated lung adenocarcinoma with KRAS mutation and TP53 alteration displayed higher global mutation rates and expressed higher levels of PD-L1 [30]. Also, some studies reported oncogene activation could induce PD-L1 expression which represents the innate immune resistance. For example, constitutive activation of NPM-ALK induced PD-L1 expression in lymphoma [31]. And up-regulation of PD-L1 expression was associated with EGFR mutation and EML4-ALK rearrangements in NSCLC [15, 16, 27]. In addition, PD-L1 expression is often induced by inflammatory factor mainly IFN- $\gamma$, which is adaptive immune resistance [18].

To explore the molecular mechanism of PD-L1 upregulation by KRAS mutation, we tested the downstream 

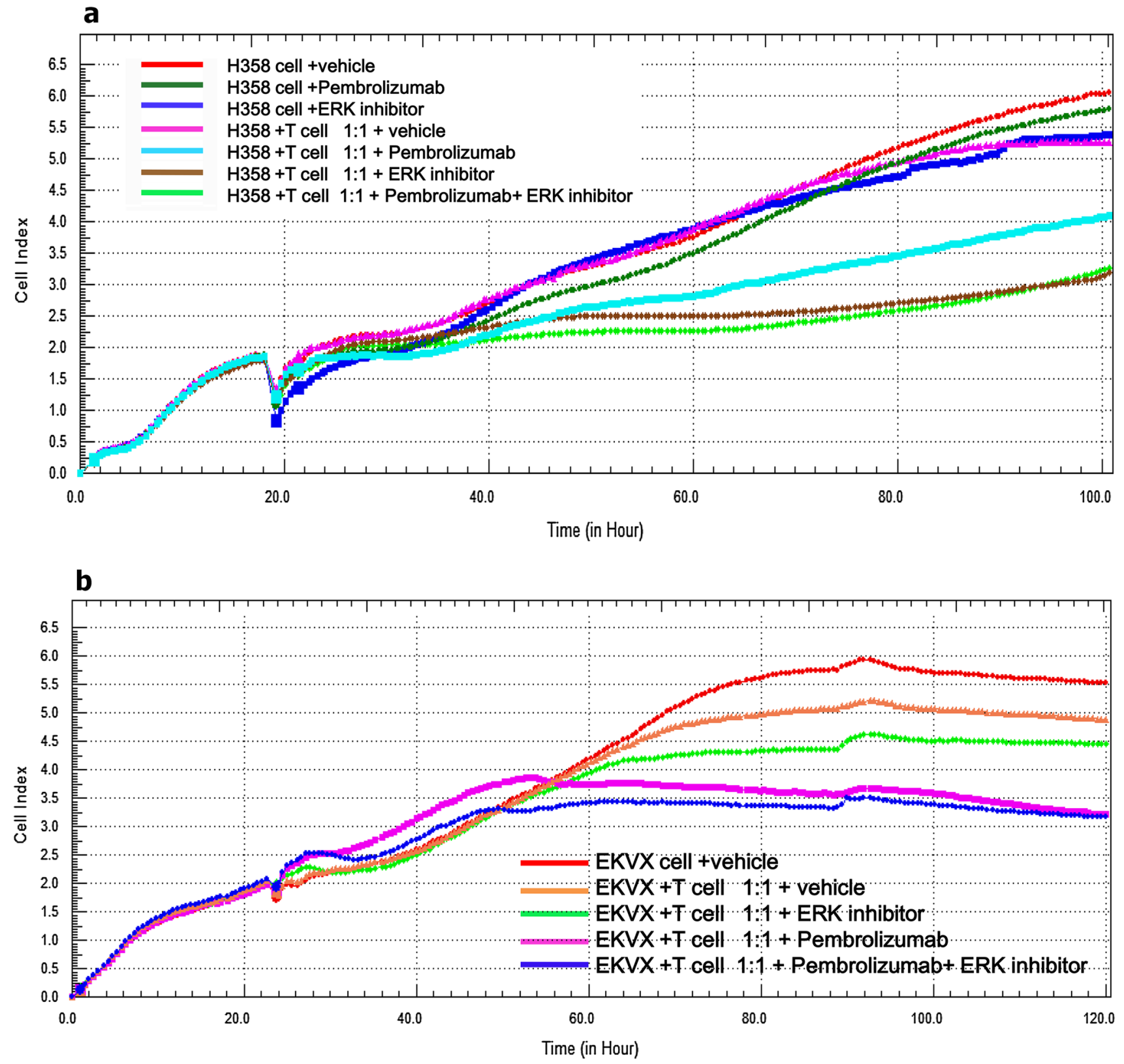

Fig. 5 The real time survival curve of KRAS mutant tumor cells (H358 and EKVX) in the co-culture system DC-CIK/H358 (a) and DC-CIK/EKVX (b) co-culture system were treated with vehicle

signaling pathways of KRAS. Mutant KRAS promotes persistent activation of downstream effectors, leading to survival and proliferation of cancer cells mainly through the Ras/Raf/MEK/ERK pathway or PI3K/AKT pathway. Our study showed that KRAS regulated PD-L1 through p-ERK but not p-AKT signaling. Our previous studies indicated that $\mathrm{p}$-ERK1/2/p-c-Jun but not p-AKT/p-S6 pathway played a critical role in remodeling the expression of PD-L1 regulated by EGFR [15].
(PBS), Pembrolizumab $(500 \mu \mathrm{g} / \mathrm{ml})$, ERK inhibitor $(100 \mathrm{nM} / \mathrm{L})$ or both. Cell index represents the cell proliferation index

P-ERK1/2/p-c-Jun pathway activation was also reported to mediate up-regulation of PD-L1 in BRAF-inhibitorresistant myeloma [32].

A previous study showed that up-regulation of PD-L1 by EGFR activation mediated the immune escape in EGFRdriven NSCLC $[15,33]$. In the present study, we found that up-regulation of PD-L1 by KRAS mutation induced the apoptosis of $\mathrm{CD} 3+\mathrm{T}$ cells through the PD-1/PD-L1 axis. $\mathrm{CD} 3+\mathrm{T}$ cells represent the major sub-population of $\mathrm{T}$ cells 
and the apoptosis of CD3+ T cells forebode the anergy and exhaustion of $\mathrm{T}$ cells, which results in the immune escape of NSCLC cells.

PD-1/PD-L1 axis is regarded as an important inhibitory pathway in the immune system that is crucial for maintaining self-tolerance and preventing excessive and potentially deleterious T-cell activity [34]. Therapies targeting PD-1 such as Pembrolizumab and Nivolumab have recently shown encouraging efficacy in specific subpopulation of patients with NSCLC [13, 35-37]. We found that Pembrolizumab could re-activate the anti-tumor immunity of $\mathrm{T}$ cells and decrease the survival rates of NSCLC cells with endogenous KRAS mutation in co-culture system. Likewise, ERK inhibitor which down-regulated the PD-L1 expression unleashed the T cells' potence to kill KRASmutant tumor cells in the co-culture system but $100 \mathrm{nM}$ ERK inhibitor itself could not affect the survival of H358 cells. We did not observe synergistic effect on killing tumor cells with combination of Pembrolizumab and ERK inhibitor in vitro co-culture system, probably because blocking PD-1 by Pembrolizumab and inhibiting PD-L1 by ERK inhibitor disrupted the same immune pathway of PD-1/ PD-L1 axis. Nevertheless, blocking PD-1/PD-L1 axis provides a promising strategy for KRAS-mutant NSCLC with PD-L1 up-regulation. Recently, Davar reported a patient with advanced, heavily pretreated KRAS-mutant lung adenocarcinoma who developed an excellent response after a single-dose of anti-PD-1 antibody (nivolumab) [38].

In conclusion, our study found PD-L1 expression is correlated with KRAS mutation in lung adenocarcinoma. Furthermore, we clarified that PD-L1 was up-regulated by KRAS over-expression through p-ERK but not p-AKT signaling. We also found KRAS-mediated up-regulation of PD-L1 induced the apoptosis of CD3 + T cells and mediated immune escape in lung adenocarcinoma cells, which could be reversed by anti-PD-1 antibody or ERK inhibitor treatment. Pembrolizumab or ERK inhibitor could recover the anti-tumor immunity of $\mathrm{T}$ cells and decrease the survival rates of KRAS-mutant NSCLC cells in co-culture system in vitro. Our study might provide a promising therapeutic option for NSCLC with KRAS mutation.

Acknowledgements We thank Prof. Liang Chen (National Institute of Biological Sciences, Beijing, China), Prof. Jianchuan Xia (Department of Biotherapy, Sun Yat-sen University Cancer Center, Guangzhou, China) and Prof. Mengfeng Li (Department of Microbiology, Zhongshan School of Medicine, Sun Yat-sen University, Guangzhou, China) for generously providing the cell lines. This work was financially supported by Chinese National Natural Science Foundation (Grant No. 81572659 and 81601991), Medical Scientific Research Fund of Guangdong (Grant No. A2016203), Medical Scientific Research Fund of Zhuhai (2015), Open project of State Key laboratory of Oncology in South China (HN2014-05), the Young Teacher Training Program of Sun Yat-Sen University (14ykpy38) and CSCOHengrui Cancer Research Fund (KY090549). The funders had no role in study design, data collection and analysis, decision to publish, or preparation of the manuscript.

\section{Compliance with ethical standards}

Conflict of interest The authors declare that they have no conflict of interest.

Open Access This article is distributed under the terms of the Creative Commons Attribution 4.0 International License (http://creativecommons.org/licenses/by/4.0/), which permits unrestricted use, distribution, and reproduction in any medium, provided you give appropriate credit to the original author(s) and the source, provide a link to the Creative Commons license, and indicate if changes were made.

\section{References}

1. Siegel RL, Miller KD, Jemal A (2015) Cancer statistics, 2015. CA Cancer J Clin 65(1):5-29. doi:10.3322/caac.21254

2. Rothschild SI (2015) Targeted therapies in non-small cell lung cancer-beyond EGFR and ALK. Cancers 7(2):930-949. doi:10.3390/cancers 7020816

3. Sholl LM, Aisner DL, Varella-Garcia M et al (2015) Multi-institutional oncogenic driver mutation analysis in lung adenocarcinoma: the lung cancer mutation consortium experience. J Thorac Oncol 10(5):768-777. doi:10.1097/jto.0000000000000516

4. Kris MG, Johnson BE, Berry LD et al (2014) Using multiplexed assays of oncogenic drivers in lung cancers to select targeted drugs. JAMA 311(19):1998-2006. doi:10.1001/jama.2014.3741

5. Leduc C, Besse B (2015) Targeted therapies in non-small cell lung cancer in 2014. Rev Mal Respir 32(2):182-192. doi:10.1016/j.rmr.2014.08.014

6. Timar J (2014) The clinical relevance of KRAS gene mutation in non-small-cell lung cancer. Curr Opin Oncol 26(2):138-144. doi:10.1097/cco.0000000000000051

7. Dearden S, Stevens J, Wu YL et al (2013) Mutation incidence and coincidence in non small-cell lung cancer: meta-analyses by ethnicity and histology (mutMap). Ann Oncol 24(9):2371-2376. doi:10.1093/annonc/mdt205

8. Sequist LV, Heist RS, Shaw AT et al (2011) Implementing multiplexed genotyping of non-small-cell lung cancers into routine clinical practice. Ann Oncol 22(12):2616-2624. doi:10.1093/ annonc/mdr489

9. Pao W, Wang TY, Riely GJ et al (2005) KRAS mutations and primary resistance of lung adenocarcinomas to gefitinib or erlotinib. PLoS Med 2(1):e17. doi:10.1371/journal.pmed.0020017

10. Pallis A, Briasoulis E, Linardou H et al (2011) Mechanisms of resistance to epidermal growth factor receptor tyrosine kinase inhibitors in patients with advanced non-small-cell lung cancer: clinical and molecular considerations. Curr Med Chem 18(11):1613-1628

11. Martin P, Leighl NB, Tsao MS et al (2013) KRAS mutations as prognostic and predictive markers in non-small cell lung cancer. J Thorac Oncol 8(5):530-542. doi:10.1097/ JTO.0b013e318283d958

12. He J, Hu Y, Hu M et al (2015) Development of PD-1/PD-L1 pathway in tumor immune microenvironment and treatment for nonsmall cell lung cancer. Sci Rep 5:13110. doi:10.1038/srep13110

13. Brahmer J, Reckamp KL, Baas P et al (2015) Nivolumab versus docetaxel in advanced squamous-cell non-small-cell lung cancer. N Engl J Med 373(2):123-135. doi:10.1056/NEJMoa1504627 
14. Lynch TJ, Bondarenko I, Luft A et al (2012) Ipilimumab in combination with paclitaxel and carboplatin as first-line treatment in stage IIIB/IV non-small-cell lung cancer: results from a randomized, double-blind, multicenter phase II study. J Clin Oncol 30(17):2046-2054. doi:10.1200/jco.2011.38.4032

15. Chen N, Fang W, Zhan J et al (2015) Upregulation of PD-L1 by EGFR activation mediates the immune escape in EGFRdriven NSCLC: implication for optional immune targeted therapy for NSCLC patients with EGFR mutation. J Thorac Oncol 10(6):910-923. doi:10.1097/jto.0000000000000500

16. Ota K, Azuma K, Kawahara A et al (2015) Induction of PD-L1 expression by the EML4-ALK oncoprotein and downstream signaling pathways in non-small cell lung cancer. Clin Cancer Res 21(17):4014-4021. doi:10.1158/1078-0432

17. Azuma K, Ota K, Kawahara A et al (2014) Association of PD-L1 overexpression with activating EGFR mutations in surgically resected nonsmall-cell lung cancer. Ann Oncol 25(10):19351940. doi:10.1093/annonc/mdu242

18. Fang W, Zhang J, Hong S et al (2014) EBV-driven LMP1 and IFN-gamma up-regulate PD-L1 in nasopharyngeal carcinoma: implications for oncotargeted therapy. Oncotarget 5(23):12189-12202

19. Zhou J, Weng D, Zhou F et al (2009) Patient-derived renal cell carcinoma cells fused with allogeneic dendritic cells elicit anti-tumor activity: in vitro results and clinical responses. Cancer Immunol Immunother 58(10):1587-1597. doi:10.1007/ s00262-009-0668-9

20. Li Y, Pan K, Liu LZ et al (2015) Sequential cytokine-induced killer cell immunotherapy enhances the efficacy of the gemcitabine plus cisplatin chemotherapy regimen for metastatic nasopharyngeal carcinoma. PLoS ONE 10(6):e0130620. doi:10.1371/ journal.pone. 0130620

21. Hong S, Chen N, Fang W et al (2016) Upregulation of PD-L1 by EML4-ALK fusion protein mediates the immune escape in ALK positive NSCLC: implication for optional anti-PD-1/PD-L1 immune therapy for ALK-TKIs sensitive and resistant NSCLC patients. Oncoimmunology 5(3):e1094598. doi:10.1080/21624 02x.2015.1094598

22. Teng Z, Kuang X, Wang J et al (2013) Real-time cell analysisa new method for dynamic, quantitative measurement of infectious viruses and antiserum neutralizing activity. J Virol Methods 193(2):364-370. doi:10.1016/j.jviromet.2013.06.034

23. Yendamuri S, Komaki RR, Correa AM et al (2007) Comparison of limited surgery and three-dimensional conformal radiation in high-risk patients with stage I non-small cell lung cancer. J Thorac Oncol 2(11):1022-1028. doi:10.1097/ JTO.0b013e 318158d4cb

24. Cao C, Zhu ZH, Yan TD et al (2013) Video-assisted thoracic surgery versus open thoracotomy for non-small-cell lung cancer: a propensity score analysis based on a multi-institutional registry. Eur J Cardiothorac Surg 44(5):849-854. doi:10.1093/ejcts/ ezt406

25. Kondo A, Yamashita T, Tamura $\mathrm{H}$ et al (2010) Interferon-gamma and tumor necrosis factor-alpha induce an immunoinhibitory molecule, B7-H1, via nuclear factor-kappaB activation in blasts in myelodysplastic syndromes. Blood 116(7):1124-1131. doi:10.1182/blood-2009-12-255125
26. Song Z, Yu X, Cheng G et al (2016) Programmed death-ligand 1 expression associated with molecular characteristics in surgically resected lung adenocarcinoma. J Transl Med 14(1):188. doi:10.1186/s12967-016-0943-4

27. Tang Y, Fang W, Zhang Y et al (2015) The association between PD-L1 and EGFR status and the prognostic value of PD-L1 in advanced non-small cell lung cancer patients treated with EGFRTKIs. Oncotarget 6(16):14209-14219

28. Calles A, Liao X, Sholl LM et al (2015) Expression of PD-1 and its ligands, PD-L1 and PD-L2, in smokers and never smokers with KRAS mutant lung cancer. J Thorac Oncol 10(12):17261735. doi:10.1097/JTO.0000000000000687

29. Mahoney KM, Sun H, Liao X et al (2015) PD-L1 antibodies to its cytoplasmic domain most clearly delineate cell membranes in immunohistochemical staining of tumor cells. Cancer Immunol Res 3(12):1308-1315. doi:10.1158/2326-6066

30. Skoulidis F, Byers LA, Diao L et al (2015) Co-occurring genomic alterations define major subsets of KRAS-mutant lung adenocarcinoma with distinct biology, immune profiles, and therapeutic vulnerabilities. Cancer Discov 5(8):860-877. doi:10.1158/2159-8290.CD-14-1236

31. Marzec M, Zhang Q, Goradia A et al (2008) Oncogenic kinase NPM/ALK induces through STAT3 expression of immunosuppressive protein CD274 (PD-L1, B7-H1). Proc Natl Acad Sci USA 105(52):20852-20857. doi:10.1073/pnas.0810958105

32. Jiang X, Zhou J, Giobbie-Hurder A et al (2013) The activation of MAPK in melanoma cells resistant to BRAF inhibition promotes PD-L1 expression that is reversible by MEK and PI3K inhibition. Clin Cancer Res 19(3):598-609. doi:10.1158/1078-0432. CCR-12-2731

33. Akbay EA, Koyama S, Carretero J et al (2013) Activation of the PD-1 pathway contributes to immune escape in EGFR-driven lung tumors. Cancer Discov 3(12):1355-1363. doi:10.1158/2159-8290.cd-13-0310

34. Pardoll DM (2012) The blockade of immune checkpoints in cancer immunotherapy. Nat Rev Cancer 12(4):252-264. doi:10.1038/nrc3239

35. Yaqub F (2015) Nivolumab for squamous-cell non-smallcell lung cancer. Lancet Oncol 16(7):e319. doi:10.1016/ s1470-2045(15)00033-9

36. Carbognin L, Pilotto S, Milella M et al (2015) Differential activity of Nivolumab, Pembrolizumab and MPDL3280A according to the tumor expression of programmed death-ligand-1 (PD-L1): sensitivity analysis of trials in melanoma, lung and genitourinary cancers. PLoS One 10(6):e0130142. doi:10.1371/journal. pone. 0130142

37. Buque A, Bloy N, Aranda F et al (2015) Trial watch: immunomodulatory monoclonal antibodies for oncological indications. Oncoimmunology 4(4):e1008814. doi:10.1080/21624 02x.2015.1008814

38. Davar D, Socinski MA, Dacic S et al (2015) Near complete response after single dose of nivolumab in patient with advanced heavily pre-treated KRAS mutant pulmonary adenocarcinoma. Exp Hematol Oncol 4:34. doi:10.1186/s40164-015-0029-7 